\title{
Dynamical evolution of the scalar condensate in heavy ion collisions
}

\author{
László P. Csernai* \\ Department of Physics, University of Bergen, 5007 Bergen, Norway; \\ KFKI Research Institute for Particle \& Nuclear Physics, P.O. Box 49, 1525 Budapest, Hungary; \\ and School of Physics and Astronomy, University of Minnesota, Minneapolis, Minnesota 55455 \\ Paul J. Ellis \\ School of Physics and Astronomy, University of Minnesota, Minneapolis, Minnesota 55455 \\ Sangyong Jeon \\ Nuclear Science Division, Lawrence Berkeley National Laboratory, Berkeley, California 94720 \\ Joseph I. Kapusta ${ }^{\S}$ \\ TH Division, CERN, CH-1211 Geneva 23, Switzerland \\ and School of Physics and Astronomy, University of Minnesota, Minneapolis, Minnesota 55455
}

(Received 4 August 1999; published 30 March 2000)

\begin{abstract}
We derive the effective coarse-grained field equation for the scalar condensate of the linear sigma model in a simple and straightforward manner using linear response theory. The dissipative coefficient is calculated consistently at tree level on the basis of the physical processes of sigma-meson decay and of thermal sigma mesons and pions knocking sigma mesons out of the condensate. The field equation is solved for hot matter undergoing either one- or three-dimensional expansion and cooling in the aftermath of a high energy nuclear collision. The results show that the time constant for returning the scalar condensate to thermal equilibrium is of order $2 \mathrm{fm} / c$.
\end{abstract}

PACS number(s): 25.75.- q, 11.10.Wx, 11.30.Qc

\section{INTRODUCTION}

Scalar condensates often appear in quantum field theories when a symmetry is spontaneously broken. Prominent examples include the Higgs condensate and the chiral condensate. The equilibrium behavior of these condensates as a function of temperature and density has been extensively studied in the context of cosmology and heavy ion collisions. However, much remains to be learned about how these condensates really evolve in out-of-equilibrium systems.

In this paper, we study the dynamical evolution of the chiral condensate in the $O(N)$ linear sigma model and apply it to the expanding matter in a high energy heavy ion collision. We recall that the sigma field $\sigma$ represents the quark condensate $\bar{q} q$ in the sense that they both have the same transformation properties for $N=4$, corresponding to two flavors of massless quarks. At high temperatures, quarks and gluons exist in a deconfined, chirally symmetric phase. At some critical temperature of order $150 \mathrm{MeV}$ a transition to a hadronic phase occurs. In this confined and symmetrybroken phase the quark condensate is nonzero. In a collision between large nuclei the beam energy must be high enough so that the matter reaches at least approximate thermal equilibrium at a temperature greater than the critical temperature. We derive and solve an effective coarse-grained equation of

\footnotetext{
*Electronic address: csernai@sentef2.fi.uib.no

${ }^{\dagger}$ Electronic address: ellis@physics.spa.umn.edu

\#Electronic address: jeon@nta2.lbl.gov

${ }^{\S}$ Electronic address: kapusta@physics.spa.umn.edu
}

motion for the chiral condensate, or mean sigma field, starting at the critical temperature and continuing down to the temperature where the system loses its ability to maintain local equilibrium and freezes out.

The equation of motion approach we espouse here was used by Linde in his pioneering work on phase transitions in relativistic quantum field theory [1], although he did not use this technique to analyze dissipative processes or fields out of equilibrium. Our derivation of a coarse-grained field equation is based on linear response theory [2], where the connection between finite temperature averages and the evolution of an observable in real physical time is clear and straightforward. There is less clarity, in our opinion, with the influence functional [3] and closely related closed time path [4] methods as used by many others in this context [5-9]. Full nonlinear response theory is certainly equivalent to both of those methods, as indeed it must be since they all describe the same physics. However, in most cases practical calculations can only be performed when the deviation from thermal equilibrium is small. This is true of the influence functional and closed time path methods as well as response theory. Still we are able to make several improvements to the most recent treatment [8] of the linear sigma model. First, the complications associated with doubling of the field variables do not arise, nor those arising from paths in the complex time plane. Second, we make a direct connection with physical response functions. It is clear that these response functions should be evaluated in the fully interacting equilibrium ensemble. Here we shall estimate the relevant response functions by including the physical processes of sigma formation and decay via two pions, and the scattering of thermal pions 
and sigma mesons from sigma mesons in the condensate. To our knowledge, the latter processes have not been studied before in this or any related context. The amplitudes for these processes are computed in tree approximation.

In Sec. II we derive the fundamental equation. In Sec. III we calculate decay and scattering contributions to the dissipative term in the coarse-grained field equation for the condensate. In Sec. IV we solve the equation of motion in the hadronic phase of a high energy heavy ion collision. Conclusions, and extensions of this paper, are discussed in Sec. V.

\section{THE FUNDAMENTAL EQUATION}

The Lagrangian of the linear $O(N)$ sigma model involves the sigma field and a vector of $(N-1)$ pion fields, $\boldsymbol{\pi}$ :

$$
\mathcal{L}=\frac{1}{2}\left(\partial_{\mu} \sigma\right)^{2}+\frac{1}{2}\left(\partial_{\mu} \boldsymbol{\pi}\right)^{2}-\frac{1}{4} \lambda\left(\sigma^{2}+\pi^{2}-f_{\pi}^{2}\right)^{2},
$$

where $\lambda$ is a positive coupling constant and $f_{\pi}$ is the pion decay constant. In the vacuum the symmetry is spontaneously broken: the sigma field acquires a vacuum expectation value of $\langle 0|\sigma| 0\rangle=f_{\pi}$, excitations of the sigma field have mass $m_{\sigma}^{2}=2 \lambda f_{\pi}^{2}$, and the pion is a Goldstone boson since we neglect explicit chiral symmetry breaking here. The symmetry is restored by a second order phase transition at the critical temperature $T_{c}^{2}=12 f_{\pi}^{2} /(N+2)$.

When one is interested in what happens at a fixed temperature at and below $T_{c}$ the sigma field is usually expressed as

$$
\sigma(\mathbf{x}, t)=v+\sigma^{\prime}(\mathbf{x}, t)
$$

where the thermal average of the sigma field at temperature $T$ is $\langle\sigma\rangle_{e q}=v$ so that $\left\langle\sigma^{\prime}\right\rangle_{e q}=0$. The equation of motion for the fluctuating component $\sigma^{\prime}$ is

$$
\ddot{\sigma}^{\prime}-\nabla^{2} \sigma^{\prime}=\lambda f_{\pi}^{2}\left(v+\sigma^{\prime}\right)-\lambda\left(v+\sigma^{\prime}\right)^{3}-\lambda\left(v+\sigma^{\prime}\right) \pi^{2} .
$$

We now allow the sigma field to be slightly out of equilibrium, and write instead

$$
\sigma(\mathbf{x}, t)=v+\sigma_{s}(\mathbf{x}, t)+\sigma_{f}(\mathbf{x}, t) .
$$

Here $\langle\sigma\rangle=v+\sigma_{s}$, where $v$ denotes the equilibrium value as before, but the deviation has been split into two pieces: a slow part $\sigma_{s}$, whose average is nonzero, and a fast part $\sigma_{f}$, whose average is zero. (Primes have been dropped for clarity of presentation.) The notation $\langle\sigma\rangle$ denotes averaging over the space-time volume chosen for coarse-graining and thus the precise division between the fast and slow parts of the field depends on the choice that is made. For example, one may include in the slow part only those Fourier components with wavenumber below some cutoff value $[10,11]$, or one may average the field fluctuations over some coarse-graining time [12]. We shall not delve into any details here, but only remark that if the results depend strongly on the coarsegraining technique then the procedure is not very useful in the given context. In any case, the slow part represents occupation of the low momentum modes by a large number of particles and so may be treated as a slowly varying classical field. The fast part is a fully quantum field. The equilibrium ensemble average of an operator $O$ is denoted by $\langle O\rangle_{e q}$ and is characterized by the temperature $T$ and the full Hamiltonian determined from the linear sigma model Lagrangian in the absence of $\sigma_{s}$. When this equilibrium ensemble is perturbed by the presence of the field $\sigma_{s}$ the Hamiltonian is modified and the resulting (nonequilibrium) ensemble average is denoted by $\langle O\rangle$.

It should be noted at this point that we have not allowed for a nonzero ensemble average of the pion field. Such a nonzero average is usually referred to as a disoriented chiral condensate, or DCC. One could certainly allow for a DCC and carry through the following computations in a straightforward way. However in thermal equilibrium a DCC never develops (although it can arise from thermal fluctuations in a small system, but even then the probability is small [13]). This is in contrast to the scalar condensate, whose value is zero above the critical temperature and becomes nonzero below it. (A very small pion mass, too small even to affect the equation of state or correlation functions at the temperatures of interest, will still tilt the system towards a unique vacuum.) Thus we choose to focus on the behavior of the $\sigma$ field.

Let us average the sigma field equation over time and length scales large compared to the scales characterizing the quantum fluctuations of the fields $\sigma_{f}$ and $\pi$, but short compared to the scales typifying $\sigma_{s}$. It is an assumption that such a separation exists, but in any given situation it can be verified or refuted a posteriori. Since $\left\langle\sigma_{f}\right\rangle=0$ we obtain

$$
\begin{aligned}
\ddot{\sigma}_{s}-\nabla^{2} \sigma_{s}= & \lambda\left(v+\sigma_{s}\right)\left[f_{\pi}^{2}-\left(v+\sigma_{s}\right)^{2}-3\left\langle\sigma_{f}^{2}\right\rangle\right. \\
& \left.-\left\langle\boldsymbol{\sigma}^{2}\right\rangle\right]-\lambda\left\langle\sigma_{f}^{3}\right\rangle .
\end{aligned}
$$

The full ensemble averages are

$$
\begin{aligned}
& \left\langle\sigma_{f}^{2}\right\rangle=\left\langle\sigma_{f}^{2}\right\rangle_{e q}+\delta\left\langle\sigma_{f}^{2}\right\rangle, \\
& \left\langle\boldsymbol{\sigma}^{2}\right\rangle=\left\langle\boldsymbol{\sigma}^{2}\right\rangle_{e q}+\delta\left\langle\boldsymbol{\sigma}^{2}\right\rangle, \\
& \left\langle\sigma_{f}^{3}\right\rangle=\left\langle\sigma_{f}^{3}\right\rangle_{e q}+\delta\left\langle\sigma_{f}^{3}\right\rangle,
\end{aligned}
$$

where the deviations are caused by $\sigma_{s}$ and are generally proportional to $\sigma_{s}$ to some positive power. Equation (5) must be satisfied even when $\sigma_{s}$ vanishes. That determines the equilibrium value of $v$ to be

$$
v=0 \quad \text { if } T>T_{c}
$$

or

$$
v^{2}=f_{\pi}^{2}-3\left\langle\sigma_{f}^{2}\right\rangle_{e q}-\left\langle\pi^{2}\right\rangle_{e q}-\left\langle\sigma_{f}^{3}\right\rangle_{e q} / v \quad \text { if } T<T_{c}
$$

We are interested in the second of these because it represents the low temperature symmetry-broken phase. To first approximation in either (i) a perturbative expansion in $\lambda$ or (ii) an expansion in $1 / N$ [14], which are the usual approximations for the sigma model, the field fluctuations are 


$$
\left\langle\sigma_{f}^{2}\right\rangle_{e q}=\int \frac{d^{3} p}{(2 \pi)^{3}} \frac{1}{E_{\sigma}} n_{B}\left(E_{\sigma} / T\right),
$$

where $E_{\sigma}=\sqrt{m_{\sigma}^{2}+p^{2}}$, and $n_{B}(E / T)=1 /(\exp (E / T)-1)$ is the Bose-Einstein distribution. Note that the sigma mass is still to be determined. The pion fluctuations are

$$
\left\langle\boldsymbol{\pi}^{2}\right\rangle_{e q}=(N-1) \frac{T^{2}}{12} .
$$

The latter follows from the fact that there are $N-1$ Goldstone bosons. Corrections to these formulas come from interactions not included in the effective mass. The sigma mass is very large at zero temperature and vanishes at $T_{c}$. The term $\left\langle\sigma_{f}^{3}\right\rangle_{e q} / v$ is not zero on account of the cubic selfcoupling of the $\sigma$ with coupling coefficient $\lambda v$. It does have a finite limit as $v \rightarrow 0$. However, once the approximations (11) and (12) have been made it is not legitimate to keep this term because it is one higher power in $\lambda$ and/or $1 / N$, and keeping it would violate the $O(N)$ symmetry. Therefore the limits are

$$
\begin{aligned}
& T \rightarrow 0: \quad v^{2}=f_{\pi}^{2}-(N-1) T^{2} / 12, \\
& T \rightarrow T_{c}: \quad v^{2}=f_{\pi}^{2}-(N+2) T^{2} / 12,
\end{aligned}
$$

where the formula for $T_{c}$ was given earlier. It is, of course, consistent with the above expression.

Using Eqs. (6)-(8) and (10) in Eq. (5), the coarse-grained field equation for $\sigma_{s}$ can now be written as

$$
\begin{aligned}
\ddot{\sigma}_{s}-\nabla^{2} \sigma_{s}+2 \lambda v^{2} \sigma_{s}= & -\lambda\left[3 v \sigma_{s}^{2}+\sigma_{s}^{3}+\delta\left\langle\sigma_{f}^{3}\right\rangle+\left(v+\sigma_{s}\right)\right. \\
& \left.\times\left(3 \delta\left\langle\sigma_{f}^{2}\right\rangle+\delta\left\langle\boldsymbol{\pi}^{2}\right\rangle\right)\right] .
\end{aligned}
$$

Note that here the system is assumed to be at a fixed temperature so that $v(T)$ is a constant. If the temperature is allowed to vary then time derivatives of $v$ must be included; see the next section. Equation (14) shows that the sigma mass and the equilibrium value of the scalar condensate are related by $m_{\sigma}^{2}=2 \lambda v^{2}$. They are temperature dependent and determined self-consistently from the formula

$$
m_{\sigma}^{2}=2 \lambda\left[f_{\pi}^{2}-(N-1) \frac{T^{2}}{12}-3 \int \frac{d^{3} p}{(2 \pi)^{3}} \frac{1}{E_{\sigma}} n_{B}\left(E_{\sigma} / T\right)\right] .
$$

The limits $T \rightarrow 0$ and $T \rightarrow T_{c}$ are readily obtained from those of $v$. An interpolating formula which connects these two limits is

$$
\frac{m_{\sigma}^{2}}{2 \lambda f_{\pi}^{2}}=\frac{v^{2}}{f_{\pi}^{2}} \approx \frac{1-T^{2} / T_{c}^{2}}{1-[3 /(N+2)]\left(T^{2} / T_{c}^{2}\right)\left(1-T^{2} / T_{c}^{2}\right)} .
$$

This is useful when studying solutions of the equation of motion numerically.

The deviations in the fluctuations due to the presence of $\sigma_{s}$ give rise to a renormalization of the parameters in the equation of motion of $\sigma_{s}$ but, more importantly, they lead to dissipation. Energy can be transferred between the field $\sigma_{s}$ and the fields $\sigma_{f}$ and $\pi$. These deviations in the fluctuations may be computed using linear response theory $[2,15]$ as long as $\sigma_{s}$ is small. Here small means in comparison to either $v$ (most relevant at low temperature) or to $\sqrt{\left\langle\sigma_{f}^{2}\right\rangle}$ (most relevant at high temperature). Technically, linear response theory is an expansion in powers of the Hamiltonian coupling the out-of-equilibrium field $\sigma_{s}$ to the other modes of the system, and guarantees that symmetries of the theory are respected. This piece of the Hamiltonian consists of positive powers of $\sigma_{s}$ and so becomes smaller and smaller with decreasing departures from equilibrium. The coupling between the slow modes and the fast modes are determined straightforwardly from the potential to be

$$
H_{s \pi}=\lambda\left(v \sigma_{s}+\frac{1}{2} \sigma_{s}^{2}\right) \pi^{2}
$$

and

$$
H_{s f}=\lambda\left[\sigma_{s}^{2}+3 v \sigma_{s}+3 v^{2}-f_{\pi}^{2}+\frac{3}{2}\left(\sigma_{s}+2 v\right) \sigma_{f}+\sigma_{f}^{2}\right] \sigma_{s} \sigma_{f} .
$$

In order to apply linear response analysis we need some initial conditions. In a nuclear collision, or in the early universe for that matter, it is assumed that the system reaches a state of thermal equilibrium at some negative time, that the system expands and cools, and at time $t=0$ the system is at the critical temperature. This implies the initial condition $\sigma_{s}(\mathbf{x}, t=0)=0$. (In a finite volume one may wish to consider an ensemble of initial values chosen from a canonical distribution [13].) From the standard theory of linear response one immediately deduces that

$$
\begin{aligned}
\delta\left\langle\sigma_{f}^{n}(x)\right\rangle= & i \lambda \int_{0}^{t} d t^{\prime} \int d^{3} x^{\prime}\left[\left(3 v^{2}-f_{\pi}^{2}\right) \sigma_{s}\left(x^{\prime}\right)+3 v \sigma_{s}^{2}\left(x^{\prime}\right)+\sigma_{s}^{3}\left(x^{\prime}\right)\right]\left\langle\left[\sigma_{f}\left(x^{\prime}\right), \sigma_{f}^{n}(x)\right]\right\rangle_{e q} \\
& +i 3 \lambda \int_{0}^{t} d t^{\prime} \int d^{3} x^{\prime}\left[v \sigma_{s}\left(x^{\prime}\right)+\frac{1}{2} \sigma_{s}^{2}\left(x^{\prime}\right)\right]\left\langle\left[\sigma_{f}^{2}\left(x^{\prime}\right), \sigma_{f}^{n}(x)\right]\right\rangle_{e q} \\
& +i \lambda \int_{0}^{t} d t^{\prime} \int d^{3} x^{\prime} \sigma_{s}\left(x^{\prime}\right)\left\langle\left[\sigma_{f}^{3}\left(x^{\prime}\right), \sigma_{f}^{n}(x)\right]\right\rangle_{e q}
\end{aligned}
$$

and 


$$
\delta\left\langle\boldsymbol{\pi}^{2}(x)\right\rangle=i \lambda \int_{0}^{t} d t^{\prime} \int d^{3} x^{\prime}\left[v \sigma_{s}\left(x^{\prime}\right)+\frac{1}{2} \sigma_{s}^{2}\left(x^{\prime}\right)\right]\left\langle\left[\boldsymbol{\pi}^{2}\left(x^{\prime}\right), \boldsymbol{\pi}^{2}(x)\right]\right\rangle_{e q} .
$$

The response functions are just the commutators of powers of the field operators evaluated at two different space-time points in the unperturbed (equilibrium) ensemble. We should emphasize that the unperturbed ensemble does include all interactions among the fast modes and makes no approximation regarding the strength of these interactions. Insertion of these deviations into Eq. (14) represents the fundamental equation of this paper. For very small departures from equilibrium it is sufficient to keep only those terms which are linear in $\sigma_{s}$. The high temperature, symmetric phase is obtained by setting $v=0$.

The time-delayed response of the fast modes to the slow one evident in Eqs. (19) and (20) has two effects: The sigma mass and self-interactions are modified, and dissipation occurs. These effects may be seen by expanding the slow field $\sigma_{s}\left(\mathbf{x}^{\prime}, t^{\prime}\right)$ in a Taylor series about the point $(\mathbf{x}, t)$ in Eqs. (19) and (20) (although such an expansion is not required). Terms with no derivative or an even number of derivatives either renormalize existing terms in the equation of motion or add new nondissipative ones, such as $\sigma_{s} \nabla^{2} \sigma_{s}$ and $\nabla^{2} \nabla^{2} \sigma_{s}$. Terms with an odd number of derivatives are explicitly dissipative. Examples are $\dot{\sigma}_{s}, \sigma_{s} \dot{\sigma}_{s}$, and $\nabla^{2} \dot{\sigma}_{s}[12]$.

Perhaps the closest analysis to ours is due to Rischke [8]. The differences may be summarized thusly: First, Rischke used the influence functional method [3], which is closely related to the closed-time-path method [4], for deriving the equations of motion of the classical field. We use linear response theory. Since the former techniques ultimately rely on a perturbative expansion in terms of $\sigma_{s}$ anyway, one might as well employ linear response theory to begin with. Linear response theory is quicker, easier to use, and more intuitive. Second, we write down the field equation for $\sigma_{s}$, which is the deviation of the scalar condensate from its equilibrium value $v$. Rischke writes down the equation of motion for $\bar{\sigma}$ $=v+\sigma_{s}$. Of course these approaches are equivalent. Third, Rischke's response functions are valid for free fields only. For example, a response function from Eqs. (19) and (20) has the form $\left\langle\left[\phi^{2}\left(\mathbf{x}^{\prime}, t^{\prime}\right), \phi^{2}(\mathbf{x}, t)\right]\right\rangle_{e q}$. For free fields this may be written $2\left[D_{>}^{2}\left(x^{\prime}-x\right)-D_{<}^{2}\left(x^{\prime}-x\right)\right]$ where the $D$ are Wightmann functions/propagators and the subscript indicates whether $t^{\prime}$ is greater than or less than $t$. Using our approach it is clear that these response functions should be evaluated in the fully interacting ensemble (but unperturbed by $\sigma_{s}$ ). Taken together with the second difference this constitutes a major improvement over Rischke's analysis. This will be discussed in more detail in the next section. Fourth, Rischke employed a particular coarse graining technique by separating soft and hard modes according to the magnitude of the momentum. We have left the coarse graining technique open. Finally, with a view towards the formation of disoriented chiral condensates, or DCC, Rischke allowed for slow classical components of the pion field. We have not done so here, mainly to keep the analysis as simple and direct as possible, although it could easily worked out in the same way as the sigma field.

\section{ESTIMATING THE RESPONSE FUNCTIONS}

One's first inclination might be to evaluate the response functions using free fields. Suppose, for example, that $\sigma_{s}$ is so slowly varying in space and time that to first approximation it can be taken outside the integration over $\mathbf{y}$ and $t^{\prime}$. Then it is a simple exercise to show that for a free field $\phi$ with mass $m$ and energy $E$ one gets

$$
\begin{aligned}
& \int_{0}^{t} d t^{\prime} \int d^{3} x^{\prime}\left\langle\left[\phi^{2}\left(\mathbf{x}^{\prime}, t^{\prime}\right), \phi^{2}(\mathbf{x}, t)\right]\right\rangle_{e q} \\
& \quad=i \int_{0}^{t} d t^{\prime} \int \frac{d^{3} p}{(2 \pi)^{3}} \frac{1}{E^{2}}\left(2 n_{B}(E / T)+1\right) \sin \left[2 E\left(t-t^{\prime}\right)\right] .
\end{aligned}
$$

The temperature-independent piece is a vacuum contribution and may be dropped for the present discussion. In the case that $m=0$ the momentum integral is done trivially with the following result:

$$
\frac{i}{4 \pi^{2}} \int_{0}^{t} d s\left[\frac{2 \pi T}{\tanh (2 \pi T s)}-\frac{1}{s}\right] .
$$

In the limit that $t$ becomes large compared to $1 / 2 \pi T$ this approaches the asymptotic value $i T t / 2 \pi$. This corresponds to the first term of a Taylor expansion of $\sigma_{s}$ and further terms in the series bring in powers of $\left(t-t^{\prime}\right)$ yielding dissipative coefficients that grow as $t^{2}, t^{3}$, and so on. This is clearly unacceptable. The origin of this problem can be traced to the inadequacy of evaluating the response functions in the free field limit. Indeed, these response functions are closely related to the shear and bulk viscosities via the Kubo formulas which express those quantities in terms of ensemble averages of commutators of the energy-momentum tensor density operator at two different space-time points. It is known that determination of the viscosities requires summation of all ladder diagrams at a minimum [16].

An alternative is to make a harmonic approximation [7] instead of a Taylor series expansion which, in this situation, can be formulated as

$$
\sigma_{s}(t-s) \approx \sigma_{s}(t) \cos \left(m_{\sigma} s\right)-\dot{\sigma}_{s}(t) \sin \left(m_{\sigma} s\right) / m_{\sigma} .
$$

For the applications we have in mind in this paper the deviation of the scalar condensate from its equilibrium value is not expected to oscillate significantly; rather, it is expected to decay exponentially to zero. Therefore the harmonic approximation does not solve the problem. 
Rather than attempt a sophisticated evaluation of the response functions in this paper we shall proceed to estimate them based on direct physical reasoning. There are two obvious mechanisms for adding or removing sigma mesons from the condensate: (1) decay into two pions or the reverse process of formation via two pion annihilation, and (2) a meson from the thermal bath elastically scattering off a sigma meson and knocking it out of the condensate. The first of these processes is included in all analyses of the linear sigma model; the second of these processes has not been studied in the literature to our knowledge. We shall evaluate them consistently at the tree level. This means that the parameters in the Lagrangian are to be fitted to experimental data also at the tree level. Let us examine each of these in turn.

The decay rate of a sigma meson into two pions in the sigma's rest frame is [17]

$$
\Gamma_{\sigma \rightarrow \pi \pi}=\frac{(N-1)}{8 \pi} \frac{\lambda^{2} v^{2}}{m_{\sigma}}=\frac{(N-1)}{16 \pi} \lambda m_{\sigma},
$$

when account is taken of the relation $m_{\sigma}^{2}=2 \lambda v^{2}$. The decay rate for a sigma meson at rest in the finite temperature system is Bose-enhanced by a factor of $\left[1+n_{B}\left(m_{\sigma} / 2 T\right)\right]^{2}$, where $n_{B}$ is the Bose-Einstein occupation number with the indicated argument of its exponential. The rate for two pions to form a sigma meson at rest is obtained from detailed balance by multiplying $\Gamma_{\sigma \rightarrow \pi \pi}$ by $n_{B}^{2}\left(m_{\sigma} / 2 T\right)$. Therefore the net rate is

$$
\begin{aligned}
\Gamma_{\sigma \pi \pi} & =\frac{(N-1)}{16 \pi} \lambda m_{\sigma}\left[\left(1+n_{B}\right)^{2}-n_{B}^{2}\right] \\
& =\frac{(N-1)}{16 \pi} \lambda m_{\sigma}\left[1+2 n_{B}\right] \\
& =\frac{(N-1)}{16 \pi} \lambda m_{\sigma} \operatorname{coth}\left(m_{\sigma} / 4 T\right) .
\end{aligned}
$$

(The argument of $n_{B}$ is the same as above.) This leads to the dissipative term $\Gamma_{\sigma \pi \pi} \dot{\sigma}_{s}$ in the equation of motion [18]. It agrees with Eq. (80) of Rischke [8].

Scattering of a thermal boson $b$ off a sigma meson with negligibly small momentum and which is considered to be a component of the background field $\sigma_{s}$ can be studied by evaluating the self-energy $[19,20]$

$$
\begin{aligned}
\Pi_{\sigma b} & =-4 \pi \int \frac{d^{3} p}{(2 \pi)^{3}} n_{B}(E / T) \frac{\sqrt{s}}{E} f_{\sigma b}^{\text {(c.m. })}(s) \\
& =-\frac{2}{\pi} \int_{m_{b}}^{\infty} d E p n_{B}(E / T) \sqrt{s} f_{\sigma b}^{\text {(c.m. })}(s) .
\end{aligned}
$$

Here $E$ and $p$ are the energy and momentum of the boson $b$, $s=m_{\sigma}^{2}+m_{b}^{2}+2 m_{\sigma} E$, and $f_{\sigma b}$ is the forward scattering amplitude. The normalization of the amplitude corresponds to the standard form of the optical theorem

$$
\sigma=\frac{4 \pi}{q_{\text {c.m. }}} \operatorname{Im} f^{(\text {c.m. })}(s),
$$

where $q_{\text {c.m. }}$ is the momentum in the c.m. frame. Because the cross section is invariant under longitudinal boosts the scattering amplitude transforms as follows:

$$
m_{\sigma} f_{\sigma b}^{(\sigma \text { rest frame })}=\sqrt{s} f_{\sigma b}^{(\text {c.m. })} .
$$

The imaginary part of the self-energy

$$
\operatorname{Im} \Pi_{\sigma b}=-\frac{m_{\sigma}}{2 \pi^{2}} \int_{m_{b}}^{\infty} d E p^{2} n_{B}(E / T) \sigma_{\sigma b}(s)
$$

determines the rate at which the field decays [18]:

$$
\Gamma_{\sigma b}=-\operatorname{Im} \Pi_{\sigma b} / m_{\sigma} .
$$

The applicability of this expression is limited to those cases where interference between sequential scatterings is negligible.

First consider pion scattering. We will calculate to tree level only and suppose that the parameters of the theory are adjusted to reproduce low energy experimental data at this same level. The invariant amplitude is

$$
\mathcal{M}=-2 \lambda\left[1+m_{\sigma}^{2}\left(\frac{1}{s}+\frac{1}{u}+\frac{3}{t-m_{\sigma}^{2}}\right)\right] .
$$

The $s, t$, and $u$ are standard Mandelstam variables satisfying $s+t+u=2 m_{\sigma}^{2}$. Note that the forward scattering amplitude evaluated at threshold, $\mathcal{M}\left(s=m_{\sigma}^{2}, t=0\right)$, vanishes in accordance with Adler's Theorem [21]. The differential cross section in the center-of-momentum frame is

$$
\frac{d \sigma}{d \Omega_{\mathrm{c} . \mathrm{m} .}}=\frac{|\mathcal{M}|^{2}}{64 \pi^{2} s}
$$

The total cross section is given by

$$
\begin{aligned}
\frac{4 \pi s}{\lambda^{2}} \sigma= & \frac{\left(s^{\prime}+1\right)^{2}}{s^{\prime 2}}+\frac{s^{\prime}}{2-s^{\prime}}+\frac{9 s^{\prime}}{s^{\prime 2}-s^{\prime}+1} \\
& -\frac{2\left(s^{\prime 2}-3 s^{\prime}-1\right)}{\left(s^{\prime}-1\right)^{3}} \ln \left[s^{\prime}\left(2-s^{\prime}\right)\right] \\
& -\frac{6\left(s^{\prime 2}-s^{\prime}-1\right)}{\left(s^{\prime}-1\right)^{3}} \ln \left[\frac{s^{\prime 2}-s^{\prime}+1}{s^{\prime}}\right],
\end{aligned}
$$

where $s$ has been scaled by the sigma mass: $s^{\prime}=s / m_{\sigma}^{2}$. The total cross section has a branch point and a pole at $s=2 m_{\sigma}^{2}$ due to the $u$ channel exchange of a sigma meson. Going beyond the tree level is necessary to incorporate the finite width of the sigma meson and regulate the singularity. Indeed, the tree approximation is not reliable at high energy. For example, it is well known that unitarity is violated in $\pi \pi$ scattering at energies of order of one to two times $m_{\sigma}$ [22]. Therefore we are only allowed to use the low energy limit, which is quite acceptable when $T \ll m_{\sigma}$. In this limit 


$$
\sigma=\frac{112}{3 \pi} \lambda^{2} \frac{p^{4}}{m_{\sigma}^{6}}
$$

where $p$ is the pion momentum in the sigma rest frame. Notice the workings of Adler's theorem here: According to that theorem the forward scattering amplitude $f$ evaluated in the rest frame of any target particle must vanish like $p^{2}$ as $p$ $\rightarrow 0$. Notice also that the total cross section cannot be obtained from the imaginary part of $f$ because we are essentially using a Born approximation. In the limit that $T \rightarrow T_{c}$ we have the opposite situation where $m_{\sigma} \ll T$. Then the three-point vertices do not contribute $[\mathcal{M}=-2 \lambda$ in place of Eq. (29)] and so use of

$$
\sigma=\frac{\lambda^{2}}{4 \pi s}
$$

may be considered more appropriate.

The contribution to the imaginary part of the self-energy is readily determined in these two limits. At low energy/ temperature

$$
\operatorname{Im} \Pi_{\sigma \pi}=-\frac{13440}{\pi^{3}} \zeta(7) \lambda^{2} \frac{T^{7}}{m_{\sigma}^{5}},
$$

and at high energy/temperature

$$
\operatorname{Im} \Pi_{\sigma \pi}=-\frac{\lambda^{2} T^{2}}{96 \pi} .
$$

These are the contributions from a single pion and must be multiplied by $N-1$ to factor in all pions. For the accuracy required in this paper we can construct a Padé approximant to represent these results:

$$
\operatorname{Im} \Pi_{\sigma \pi} \approx-(N-1) \frac{\lambda^{2} T^{2}}{96 \pi} \frac{T^{5}}{\left[T^{5}+\left(m_{\sigma} / 10.568\right)^{5}\right]} .
$$

Notice that this contribution to $\Gamma$ diverges as $T \rightarrow T_{c}$ on account of division by $m_{\sigma}$. This ought to come as no surprise since one is approaching a critical point where certain fluctuations diverge.

The above analysis may be repeated for a sigma meson knocking another out of the condensate. The invariant amplitude for $\sigma \sigma \rightarrow \sigma \sigma$ is

$$
\mathcal{M}=-6 \lambda\left[1+3 m_{\sigma}^{2}\left(\frac{1}{s-m_{\sigma}^{2}}+\frac{1}{u-m_{\sigma}^{2}}+\frac{1}{t-m_{\sigma}^{2}}\right)\right]
$$

reflecting the symmetry in the $s, t, u$ channels. The total cross section is

$$
\begin{aligned}
\frac{8 \pi s}{9 \lambda^{2}} \sigma= & \left(\frac{s^{\prime}+2}{s^{\prime}-1}\right)^{2}+\frac{18}{s^{\prime}-3}-\frac{12\left(s^{\prime 2}-3 s^{\prime}-1\right)}{\left(s^{\prime}-4\right)\left(s^{\prime}-2\right)\left(s^{\prime}-1\right)} \\
& \times \ln \left(s^{\prime}-3\right) .
\end{aligned}
$$

This expression has no singularities because $s^{\prime} \geqslant 4$. The low energy limit is

$$
\sigma=\frac{9}{2 \pi} \frac{\lambda^{2}}{m_{\sigma}^{2}}
$$

which gives rise to the low temperature limit

$$
\operatorname{Im} \Pi_{\sigma \sigma}=-\frac{9}{2 \pi^{3}} \lambda^{2} T^{2} e^{-m_{\sigma} / T} .
$$

In the high energy limit only the four-point vertex contributes, giving

$$
\sigma=\frac{9}{8 \pi} \frac{\lambda^{2}}{s}
$$

which gives rise to the high temperature limit

$$
\operatorname{Im} \Pi_{\sigma \sigma}=-\frac{3}{64 \pi} \lambda^{2} T^{2}
$$

This expression is identical to that calculated by Jeon [16] and Rischke [8] in a symmetric $\phi^{4}$ model to which it can be compared. The two limits can be combined in a Padé approximant as

$$
\operatorname{Im} \Pi_{\sigma \sigma} \approx-\frac{9}{2 \pi} \frac{\lambda^{2} T^{2}}{\left[96+\pi^{2}\left(e^{m_{\sigma} / T}-1\right)\right]},
$$

which is useful for numerical computations.

The total rate is obtained by addition of all components, namely

$$
\Gamma=\Gamma_{\sigma \pi \pi}+\Gamma_{\sigma \pi}+\Gamma_{\sigma \sigma}
$$

where

$$
\Gamma_{\sigma \pi}=-\operatorname{Im} \Pi_{\sigma \pi} / m_{\sigma}
$$

and

$$
\Gamma_{\sigma \sigma}=-\operatorname{Im} \Pi_{\sigma \sigma} / m_{\sigma} .
$$

Other works, such as [8], do not include the latter two scattering contributions arguing that they are of order $\lambda^{2}$, while $\Gamma_{\sigma \pi \pi}$ is of order $\lambda$. While this is correct at low $T$ when $m_{\sigma}$ is large, it becomes questionable near to the critical temperature where $m_{\sigma}$ is small (see the discussion of Fig. 1 below).

It should be noted that the scattering contributions diverge at the critical temperature on account of division by the vanishing sigma mass. This may be a signal of the breakdown of the use of tree-level scattering amplitudes and requires further investigation.

\section{SOLUTION IN AN EXPANDING FIREBALL}

In this section we shall study solutions to the coarsegrained field equation in several limits. First, suppose that the volume and temperature are fixed in time but that the system is slightly out of equilibrium in the sense that $\sigma_{s}$ $\neq 0$. The field equation is then

$$
\ddot{\sigma}_{s}+\Gamma(T) \dot{\sigma}_{s}+m_{\sigma}^{2}(T) \sigma_{s}=-\lambda\left(3 v(T) \sigma_{s}^{2}+\sigma_{s}^{3}\right),
$$




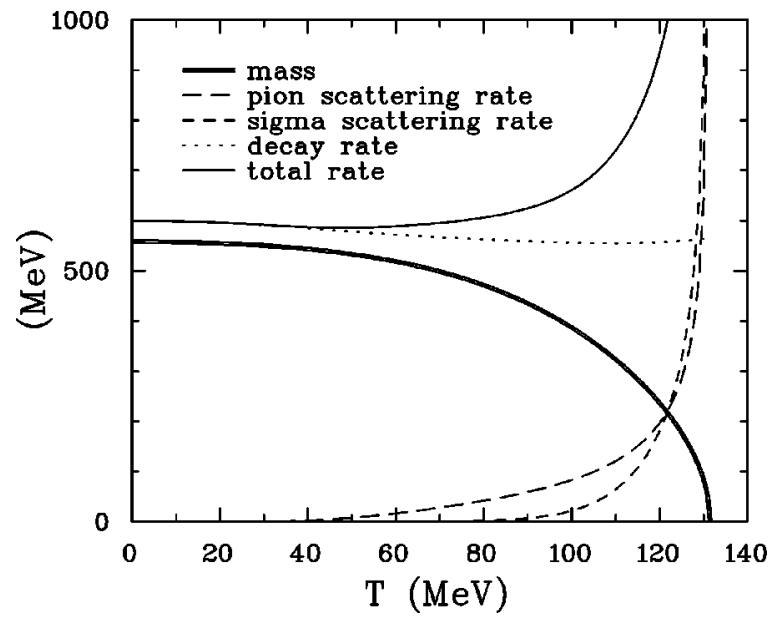

FIG. 1. The sigma mass and the decay and scattering contributions to the width of the sigma meson as functions of temperature from 0 to $T_{c}$.

where $\Gamma$ is the sum of decay and scattering terms as given in the previous section. When $\left|\sigma_{s}\right| \ll v$ this equation can be linearized. It is equivalent to a simple damped harmonic oscillator. The system is underdamped if $m_{\sigma}>\Gamma / 2$ and overdamped if $m_{\sigma}<\Gamma / 2$. We choose $\lambda=18$ so that the sigma mass in vacuum is $6 f_{\pi}$, corresponding to the $s$-wave resonance observed in the $\pi \pi$ channel. In Fig. 1 we plot $m_{\sigma}$ and the individual contributions to $\Gamma$ as functions of temperature $T$. The field is overdamped when $T>0.8 T_{c}$.

Next consider the expansion of the system created in a high energy nuclear collision. If the beam energy is high enough it will form a quark-gluon plasma with temperature greater than $T_{c}$. This "fireball" will expand and cool, eventually reaching $T_{c}$. At this moment, say at time $t=t_{c}$, the initial conditions for the coarse-grained field must be specified. We will assume, for the sake of illustration, that the system is locally uniform so that spatial gradients are unimportant. Depending on whether the system is expanding spherically or only longitudinally along the beam axis $(D$ $=3$ or 1 , respectively), we obtain the modified equation of motion

$$
\begin{aligned}
\ddot{\sigma}_{s} & +\ddot{v}+\frac{D}{t}\left(\dot{\sigma}_{s}+\dot{v}\right)+\Gamma(T) \dot{\sigma}_{s}+m_{\sigma}^{2}(T) \sigma_{s} \\
& =-\lambda\left(3 v(T) \sigma_{s}^{2}+\sigma_{s}^{3}\right) .
\end{aligned}
$$

In this situation $t$ is really the local, or proper, time and the term proportional to $D / t$ may be thought to arise from either the d'Alembertian or from a volume dilution term $[\dot{V}(t) / V(t)] \dot{\sigma}_{s}[23,24]$ analogous to the Hubble expansion [25]. Perhaps the easiest way to obtain this equation is to derive the equation of motion for the total condensate $\bar{\sigma}$ $=v+\sigma_{s}$ and then make the substitution. Most authors actually solve the equation of motion for $\bar{\sigma}$, but this is a matter of taste. Note that $v(T(t))$ is the instantaneous value of the equilibrium condensate and that is why no potential for it appears above.

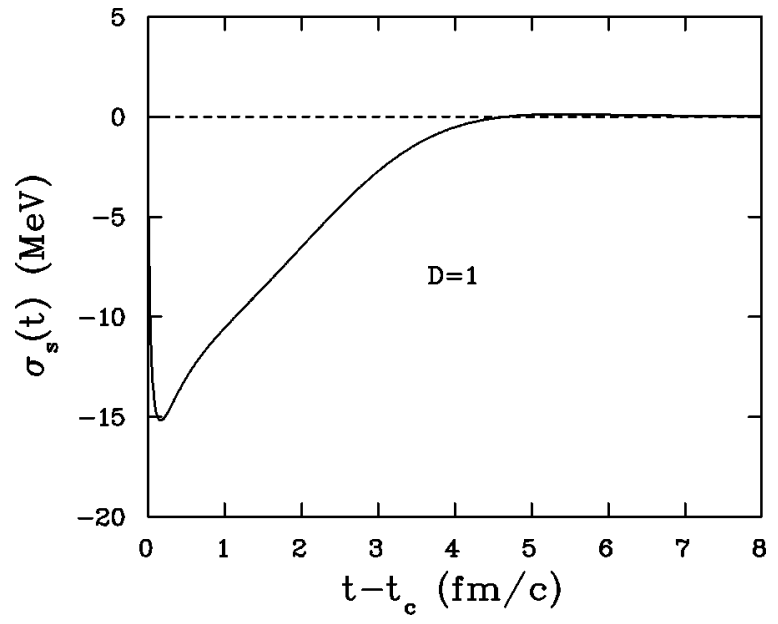

FIG. 2. The temporal evolution of the deviation of the scalar condensate from its equilibrium value $v$ in units of $\mathrm{MeV}$ for a one-dimensional expansion of hot matter produced in a high energy nuclear collision.

In addition to the coarse-grained field equation one needs to know how the local temperature evolves with time. It must be assumed that it changes slowly enough so that local equilibrium can be maintained by the rapidly fluctuating pion and sigma fields. In principle one ought to solve the equation

$$
d e / d t=-D w / t
$$

where $e$ is the energy density, $w=e+P$ is the enthalpy, and $P$ is the pressure. Rather than working out a detailed description of the equation of state, which is really dominated by the rapidly fluctuating thermal fields, we simply assume a free massless gas of pions where the pressure is proportional to $T^{4}$. The sigma meson has a mass small compared to $T$ only very near the critical temperature, and its effect is therefore generally unimportant. As a consequence, the temperature falls with time according to the law

$$
T(t)=T_{c}\left(\frac{t_{c}}{t}\right)^{D / 3} .
$$

A reasonable numerical value for $t_{c}$ is $3 \mathrm{fm} / c$ [26-29]. The back reaction of $\sigma_{s}$ on the time evolution of the temperature is thereby neglected. This is a quite reasonable approximation because very little of the total energy resides in the field $\sigma_{s}$.

The equation of motion may be solved by numerically evolving an analytic solution in the neighborhood of $t_{c}$. As $t \rightarrow t_{c}$ the equilibrium condensate $v \rightarrow 0$, however Eqs. (16) and (50) indicate that the $\ddot{v}$ and $\dot{v}$ are singular, as are the scattering contributions to the width $\Gamma$. The analytic behavior of $\sigma_{s}$ as $t \rightarrow t_{c}$ is uniquely determined by requiring that the derivatives of $\sigma_{s}$ exactly cancel these singularities, while $\sigma_{s} \rightarrow 0$. The result is displayed in Fig. 2 for $D=1$ and in Fig. 3 for $D=3$. The deviation from equilibrium $\sigma_{s}$ is maximal less than $1 / 2 \mathrm{fm} / c$ after the critical temperature is passed, and it dies away with a time scale of about $2 \mathrm{fm} / c$. There is a hint of oscillatory motion in the solutions, but basically they are overdamped. 


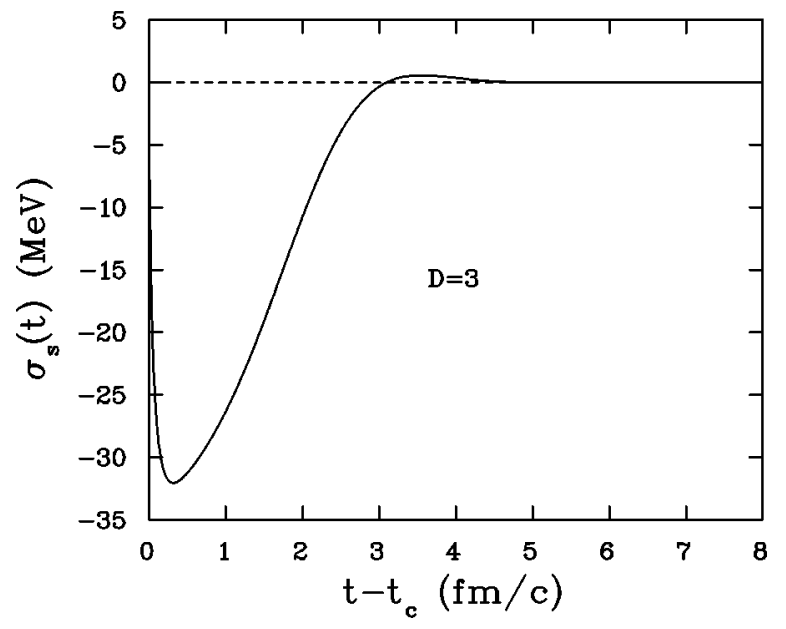

FIG. 3. The temporal evolution of the deviation of the scalar condensate from its equilibrium value $v$ in units of $\mathrm{MeV}$ for a three-dimensional expansion of hot matter produced in a high energy nuclear collision.

\section{CONCLUSION}

In this paper we have studied the dynamical evolution of the scalar condensate in the $O(N)$ linear sigma model in out-of-equilibrium situations. Our method is based on the equation of motion. Dissipation arises because of the response of the correlation functions of the fast modes to the slow modes of the fields. This is treated with standard linear response theory. These response functions should be computed exactly and used in the resulting dissipative, coarsegrained equation of motion. However, such explicit computations are generally not possible to do. Therefore, we identified the physical mechanisms responsible for the dissi- pation and estimated the corresponding response functions based on a consistent tree-level analysis. These mechanisms include the decay of sigma mesons in the condensate, and the knockout of sigma mesons in the condensate due to scattering with thermal sigma mesons and pions. To our knowledge, the latter physical mechanisms have not been studied before.

We then studied the dynamical evolution of the condensate in heavy ion collisions, after the phase transition from quark-gluon plasma to hadrons, and allowing for either one or three dimensional expansion of the hot matter. These showed that thermal equilibrium was reestablished rather rapidly, with a time constant of order $2 \mathrm{fm} / c$. Clearly, much more could be studied along these same lines, including the formation and fate of disoriented chiral condensates (DCC).

The method we used in this paper is very general, and may be applied to other theories, including nuclear matter, QCD, and electroweak theory. Such work is underway.

\section{ACKNOWLEDGMENTS}

J.K. thanks the Institute of Technology at the University of Minnesota for granting a single quarter leave in the spring of 1999 and the Theory Division at CERN for hospitality and support during that time. S.J. was supported by the Director, Office of Energy Research, Office of High Energy and Nuclear Physics, Division of Nuclear Physics, and by the Office of Basic Energy Sciences, Division of Nuclear Sciences, of the U.S. Department of Energy under Contract No. DE-AC03-76SF00098. This work was also supported by the Department of Energy under Grant No. DE-FG0287ER40328, by the NSF under travel Grant No. INT9602108 and by the Norwegian Research Council.
[1] A. D. Linde, Rep. Prog. Phys. 42, 389 (1979).

[2] A. L. Fetter and J. D. Walecka, Quantum Theory of ManyParticle Systems (McGraw-Hill, New York, 1971).

[3] R. Feynman and F. Vernon, Ann. Phys. (N.Y.) 24, 118 (1963).

[4] J. Schwinger, J. Math. Phys. 2, 407 (1961); L. V. Keldysh, Zh. Eksp. Teor. Fiz. 47, 1515 (1964) [Sov. Phys. JETP 20, 1018 (1964)].

[5] M. Gleiser and R. O. Ramos, Phys. Rev. D 50, 2441 (1994).

[6] D. Boyanovsky, H. J. de Vega, R. Holman, D. S. Lee, and A. Singh, Phys. Rev. D 51, 4419 (1995).

[7] C. Greiner and B. Müller, Phys. Rev. D 55, 1026 (1997).

[8] D. H. Rischke, Phys. Rev. C 58, 2331 (1998).

[9] C. Greiner and S. Leupold, Ann. Phys. (N.Y.) 270, 328 (1998).

[10] D. Bödeker, L. McLerran, and A. Smilga, Phys. Rev. D 52, 4675 (1995).

[11] F. Lombardo and F. D. Mazzitelli, Phys. Rev. D 53, 2001 (1996).

[12] L. P. Csernai, S. Jeon, and J. I. Kapusta, Phys. Rev. E 56, 6668 (1997)

[13] T. S. Biró, D. Molnár, Z. H. Feng, and L. P. Csernai, Phys. Rev. D 55, 6900 (1997); D. Molnár, L. P. Csernai, and Zs. Lázár, ibid. 58, 114018 (1998).
[14] A. Bochkarev and J. Kapusta, Phys. Rev. D 54, 4066 (1996); S. Jeon and J. Kapusta, ibid. 54, 6475 (1996).

[15] J. I. Kapusta, Finite Temperature Field Theory (Cambridge University, Cambridge, England, 1989).

[16] S. Jeon, Phys. Rev. D 52, 3591 (1995).

[17] B. D. Serot and J. D. Walecka, Adv. Nucl. Phys. 16 (1986).

[18] Although well known it is nevertheless worthwhile reminding ourselves how the self-energy is related to a dissipative term in a field equation. A wave of small amplitude evolves in time according to the pole of its propagator, which is determined by the zeros of $E^{2}-p^{2}-m^{2}-\Pi(E, p)$, where $\Pi(E, p)$ is the retarded self-energy. Generally $\Pi$ has both real and imaginary parts. Defining $\Gamma(p)=-\operatorname{Im} \Pi\left(E_{R}(p), p\right) / E_{R}(p)$, where $E_{R}(p)$ is the real part of the pole, one finds that the wave decays like $\exp (-\Gamma(p) t / 2)$. The origin of the factor of 2 is that $\Gamma$ represents the loss rate for the number density whereas $\Gamma / 2$ is the rate for the amplitude. See, for example, H. A. Weldon, Phys. Rev. D 28, 2007 (1983). This physics may be represented in a real Klein-Gordon wave equation by the dissipative term $\Gamma \dot{\phi}$.

[19] E. V. Shuryak, Nucl. Phys. A533, 761 (1991).

[20] V. L. Eletsky and J. I. Kapusta, Phys. Rev. C 59, 2757 (1999).

[21] S. Adler, Phys. Rev. 139, B1638 (1965). 
[22] J. L. Basdevant and B. W. Lee, Phys. Lett. 29B, 437 (1969); Phys. Rev. D 2, 1680 (1970).

[23] S. Gavin and B. Müller, Phys. Lett. B 329, 486 (1994).

[24] J. Randrup, Phys. Rev. D 55, 1188 (1997).

[25] E. W. Kolb and M. S. Turner, The Early Universe (AddisonWesley, Redwood City, CA, 1990).

[26] L. P. Csernai and J. I. Kapusta, Phys. Rev. Lett. 69, 737
(1992).

[27] L. P. Csernai, J. I. Kapusta, G. Kluge, and E. E. Zabrodin, Z. Phys. C 58, 453 (1993).

[28] L. P. Csernai and I. N. Mishustin, Phys. Rev. Lett. 74, 5005 (1995).

[29] T. S. Biró and C. Greiner, Phys. Rev. Lett. 79, 3138 (1997). 